\title{
Comparações entre práticas educativas de professores, habilidades sociais e problemas de comportamento de alunos
}

\section{Comparisons between teachers' educational practices, social skills and students' problem behavior}

\section{Comparaciones entre prácticas educativas de profesores, habilidades sociales y conducta problema de alumnos}

\section{Marília Mariano*}

Universidade Estadual Paulista "Júlio de Mesquita Filho" - UNESP, Bauru, São Paulo, Brasil

\author{
Alessandra Turini Bolsoni-Silva** \\ Universidade Estadual Paulista "Júlio de Mesquita Filho" - UNESP, Bauru, São Paulo, \\ Brasil
}

\begin{abstract}
RESUMO
As interações sociais que ocorrem no ambiente escolar podem favorecer ou prejudicar o desenvolvimento acadêmico e comportamental. Objetivou-se neste estudo comparar práticas educativas de professores e comportamentos infantis, de crianças diferenciadas por indicadores diagnósticos de problemas de comportamento (grupos clínicos e não clínicos para problemas de comportamento), pela escolaridade (pré-escolares e escolares) e sexo. A amostra foi composta por 283 crianças e seus professores da rede pública de ensino, que responderam questionários e entrevista semiestruturada. Foram conduzidas análises estatísticas: testes t de Student e Qui-quadrado. Notou-se que professores tendem a ser menos habilidosos e mais punitivos com meninos, escolares, e crianças clínicas para problemas de comportamento. Meninas e crianças não clínicas para problemas de comportamento apresentam maiores médias de habilidades sociais e menores escores de problemas comportamentais.
\end{abstract}

Palavras-chave: Práticas educativas, habilidades sociais, problemas de comportamento.

\begin{abstract}
The social interactions that occur in the school environment can favor or harm the academic and behavioral development. The objective of this study was to compare teachers' educational practices and infant behaviors of children differentiated by diagnostics indicators of behavior problems (clinical and non-clinical groups to behavior problems), by schooling (preschoolers and schoolchildren) and sex. The sample comprised 283 children and their public schools teachers, that answered questionnaires and semi-structured interview. Statistical analyses were conducted: Student t
\end{abstract}


and Chi-square tests. It was noted that teachers tend to be less skilled and more punitive with boys, schoolchildren and clinical children to behavior problems. Girls and non-clinical children to behavior problems present higher average of social skills and less scores of behavioral problems.

Keywords: Educational practices, social skills, behavior problems.

\section{RESUMEN}

Las interaciones sociales que ocurren en el ambiente escolar pueden favorecer o perjudicar el desenvolvimiento académico y comportamental. Se ha objetivado en este estudio comparar prácticas educativas de profesores y comportamientos infantiles de niños diferenciados por indicadores diagnósticos de problemas conductuales (grupos clínicos y no clínicos para problemas conductuales), por la escolaridad (preescolares y escolares) y sexo. La muestra fue compuesta por 283 niños y sus profesores de la red pública de enseñanza, que respondieron cuestionarios y entrevista semiestructurada. Fueron efectuados análisis estadísticos: tests t de Student y Chi-cuadrado. Se ha notado que profesores tienden a ser menos hábiles y más punitivos con chicos, escolares y niños clínicos para problemas conductuales. Chicas y niños no clínicos para problemas conductuales presentan mayores medias de habilidades sociales y menores escores de problemas conductuales.

Palabras clave: Prácticas educativas, habilidades sociales, problemas conductuales.

\section{I ntrodução}

Problemas de comportamento e habilidades sociais parecem ser produtos das interações sociais, que ocorrem desde a infância até a adultez, e vão se alterando conforme as exigências ambientais (Del Prette \& Del Prette, 2008; Patterson, Reid \& Dishion, 1992). Problemas de comportamento podem ser classificados em externalizante (desobediência, mentira, agressividade e roubo) ou internalizantes (tristeza, choro excessivo, isolamento social, retraimento) (Achenbach \& Edelbrock, 1978; Pedrini \& Frizo, 2010) e ocorrem em paralelo a um déficit na aquisição de habilidades sociais (Bolsoni-Silva \& Carrara, 2010; Patterson, Degarmo \& Knutson, 2000). Habilidades sociais denominam os comportamentos que contribuem para a qualidade e efetividade das interações que ocorrem entre os indivíduos (Bolsoni-Silva \& Carrara, 2010; Del Prette \& Del Prette, 2008; Golçalvez \& Murta, 2008). Respostas socialmente habilidosas, contrárias aos problemas de comportamento, parecem produzir reforçadores sociais e favorecer o desenvolvimento (Bolsoni-Silva \& Carrara, 2010; O'Connor, Dearing $\&$ Collins, 2011; Silver, J effrey, J effrey \& Marilyn, 2010).

No ambiente escolar, estudos (Baker, Grant \& Morlock, 2008; Bolsoni-Silva \& Mariano, 2014; Doumen, Verschueren, Buyse, Germeijs, Luyckx \& Soenens, 2008; Henricsson \& Rydell, 2006; O'Connor et al., 2011; Silver et al., 2010) demonstram que as 
interações entre alunos e professores também podem influenciar positivamente ou negativamente o desenvolvimento das crianças. As práticas educativas negativas (como gritar, punir, expulsar o aluno) são mais frequentes em alunos clínicos para problemas de comportamento e tendem a estimular a agressividade dos alunos, ao invés de minimizá-la (Baker, 2006; Baker et al., 2008; Bolsoni-Silva \& Mariano, 2014; Henricsson \& Rydell, 2006; Doumen et al., 2008; Silver et al., 2010). Em contrapartida, as habilidades sociais educativas e as interações afetuosas e próximas entre professores e alunos tendem a promover as habilidades sociais infantis e 0 desenvolvimento (Bolsoni-Silva \& Mariano, 2014; Henricsson \& Rydell, 2006), produzindo até mesmo um efeito compensatório nos prejuízos acarretados pelos problemas comportamentais (Baker, 2006; Silver et al., 2010).

Há ainda estudos (Buyse, Verschueren, Doumen, Damme \& Maes, 2008; Hamre \& Pianta, 2005) que discutem que com a evolução dos níveis educacionais é possível que os problemas de comportamento também progridam, já que os ambientes vão se tornando mais complexos e exigentes socialmente e academicamente. O que demonstra coerência com os parâmetros curriculares brasileiros que possuem diferentes orientações para a pré-escola (Educação Infantil - El - destinada a crianças de 4 a 6 anos) e para o período escolar (Ensino Fundamental - EF - destinado a crianças de 6 a 12 anos): a El possui como eixos norteadores, na proposta curricular, as interações sociais e brincadeiras, que propiciam a aprendizagem da cultura, o convívio em um espaço coletivo e experiências concretas da vida cotidiana (MEC, 2009); o currículo do EF direciona-se à alfabetização e o letramento, desenvolvimento de diversas formas de expressão (o aprendizado da Língua Portuguesa, a Literatura, a Música e demais artes), assim como o aprendizado de Educação Física, Matemática, Ciência, História e Geografia (MEC, 2010). Para Bolsoni-Silva et al. (2010) na El o ambiente é propício para interações e práticas lúdicas; contudo, no EF os alunos permanecem mais tempo em salas de aula, num enquadramento definido, em que provavelmente professores e crianças possuam menores oportunidades de interações, já que há destaque para as atividades acadêmicas e a criança tende a ficar mais exposta a situações de conflito (Bolsoni-Silva et al., 2010; Castro \& Bolsoni-Silva, 2008). Assim, crianças com défits comportamentais podem encontrar maiores dificuldades em se comportar no EF, o que explicaria uma progressão de frequência ou intensidade de comportamentos problema. No entanto, outros autores (Bolsoni-Silva, Marturano \& Freiria, 2010; Henricsson \& Rydell, 2006; Pizato, Marturano \& Fontaine, 2014) discutem que, no ambiente escolar, os comportamentos problema podem diminuir devido à aprendizagem de comportamentos alternativos, como habilidades sociais. 
Outra variável que interfere nos repertórios das crianças e nas relações que elas estabelecem com professores é o sexo. Alguns estudos (Baker, 2006; Henricsson, Rydell, 2006; Pizato et al., 2014; Silver et al., 2010) identificaram nas meninas escores mais altos de habilidades sociais e mais baixos de problemas de comportamento. Parece que meninas estabelecem relações de proximidade com professores, e meninos interações conflituosas, impactando na adaptação ao ambiente escolar e no desempenho social (Baker, 2006; Henricsson \& Rydell, 2006; Silver et al., 2010). Em Baker (2006) observou-se que as experiências escolares vivenciadas pelas meninas, dada a maior frequência de relações de proximidade, afeto, e os baixos índices de conflito com professores e pares, impactou positivamente o desenvolvimento. Desta forma, as meninas parecem mais disponíveis e atentas para observar os comportamentos presentes nas interações, o que pode, inclusive, favorecer a aprendizagem de habilidades sociais (Baker et al., 2008; BolsoniSilva et al., 2010). Os meninos, por sua vez, tendem a entrar na escola com mais energia, entusiasmo, agressividade e inferior desenvolvimento de maturidade, tendendo a promover mais interações de conflito com os professores e afetando a harmonia do relacionamento (Baker, 2006).

Em relação aos professores, alguns autores (Bolsoni-Silva, Mariano, 2014; Del Prette \& Del Prette, 2008) caracterizam como habilidades sociais educativas os comportamentos dos docentes que promovem aprendizagens relevantes para o desenvolvimento das crianças, como iniciar conversas, elogiar, orientar. Em contrapartida, práticas educativas negativas referem-se aos comportamentos que podem estimular a agressividade nos alunos e produzir relações de conflito (Bolsoni-Silva, Mariano, Loureiro, Bonarccorsi, 2013; Bolsoni-Silva, Mariano, 2014). Pesquisas demonstraram que educadores tendem a utilizar, em maior frequência, práticas negativas (gritar, punir, castigar) nas interações com alunos com problemas de comportamento e meninos, o que pode intensificar os problemas, ao invés de minimizá-los (Bolsoni-Silva et al., 2013; Bolsoni-Silva, Mariano, 2014; Picado \& De Rose, 2009). É possível que professores utilizem práticas negativas uma vez que a punição tende a suprimir de maneira imediata um comportamento considerado como inadequado (Sidman, 1995; Viecili \& Medeiros, 2002). No entanto, esse recurso tende a gerar efeitos colaterais, como contra-controle (fuga e esquiva) e sentimentos indesejáveis (ansiedade e medo) (Sidman, 1995; Viecili \& Medeiros, 2002). E diante de estímulos aversivos dos professores, alunos tendem a emitir comportamentos como atrasos, conversas com o colega, ficar indiferente às explicações, agressões verbais e evasão escolar (Zanotto, 2000).

$\mathrm{Na}$ revisão de literatura PSI identificaram-se estudos com a população brasileira que analisam (1) problemas de comportamento 
e/ou habilidades sociais infantis, em um dado período escolar (Bolsoni-Silva et al., 2010; Pedrini \& Frizzo, 2010; Pereira, Marturano, Gardinal-Pizato \& Fontaine, 2011; Pizato et al., 2014; Saud \& Tonelotto, 2005), (2); problemas de comportamento e/ou habilidades sociais infantis associando-os com os repertórios de professores mas em uma amostra reduzida (Bolsoni-Silva \& Mariano, 2014; Picado \& De Rose, 2009); ou ainda (3) os problemas de comportamento e sofrimento psíquico de professores (Lyra, Assis, Njaine, Oliveira \& Pires., 2009; Pas, Bradshaw, Hershfeldt \& Leaf, 2010). Assim, este estudo avança ao propor uma análise dos comportamentos dos professores e dos alunos (comportamentos problema e habilidades sociais), em diferentes sexos e períodos de escolarização, utilizando instrumentos empiricamente testados e uma amostra ampla. É relevante promover estudos com a população brasileira que aprofundem na investigação do tema para produção de dados empíricos para o desenvolvimento de futuras intervenções breves, eficazes e eficientes (Duarte \& Bordin, 2000; Pesce, 2009). Diante dessas ponderações, o presente estudo objetivou comparar habilidades sociais educativas e práticas educativas negativas de professores, habilidades sociais e problemas de comportamento, de crianças diferenciadas por indicadores diagnósticos de problemas de comportamento (grupos clínico e não clínico para problemas de comportamento), escolaridade (pré-escolares e escolares) e sexo (meninos e meninas).

\section{Método}

\subsection{Participantes}

A amostra foi composta por 283 crianças, de ambos os sexos (169 eram meninos e 114 meninas), e os respectivos professores, da Rede de Ensino Público de uma cidade do Centro-Oeste do Estado de São Paulo. Na pré-escola: (1) participantes: 113 crianças; (2) sexo das crianças: 56 meninos e 57 meninas; (3) idade das crianças: variação entre três e seis anos, com média de 3,98 e desvio padrão 0,$90 ;(4)$ idade dos docentes: variação entre 26 e 60 anos, com média igual a 43 e desvio padrão de 5,92; (5) escolaridade dos docentes: maioria (107) declarou que concluiu o curso superior, quatro professores possuíam curso superior incompleto e dois concluíram apenas o ensino médio; (6) sexo dos docentes: um professor era do sexo masculino; (7) turnos de trabalho: 84 docentes lecionavam em uma escola, enquanto que 28 trabalhavam em duas escolas e um professor em três escolas. No período escolar: (1) participantes: 170 crianças; (2) sexo das crianças: 113 meninos e 57 meninas; (3) idade das crianças: variação entre 6 e 12 anos, média da idade foi 
8,33 e desvio padrão 1,70 ; (4) idade dos docentes: variação entre 22 e 64 anos, com média igual a 36 e desvio padrão de 7,99; (5) escolaridade dos docentes: todos concluíram o curso superior e dois possuíam pós-graduação; (6) sexo dos docentes: 13 professores eram do sexo masculino; (7) turnos de trabalho: 63 docentes lecionavam em uma escola, enquanto que 99 trabalhavam em duas escolas e oito em três escolas.

\subsection{I nstrumentos}

Para cumprir com os objetivos proposto, foram utilizados três instrumentos: Teacher's Report Form (TRF) (Achenbach \& Rescorla, 2001); Roteiro de Entrevista de Habilidades Sociais Educativas para Professores - RE-HSE-Pr - Adaptação feita por Mariano (2011); Questionário de Respostas Socialmente Habilidosas (versão para professores) - QRSH-Pr (Bolsoni-Silva, Marturano \& Loureiro, 2009). O TRF consiste em uma lista de verificação comportamental de crianças e adolescentes, a partir das avaliações dos professores, resultando na classificação de categorias clínica, limítrofe ou não clínica para problema de comportamento. O TRF é uma avaliação mundialmente utilizada para identificar problemas de saúde mental, empiricamente testada, traduzido e retrotraduzido para o português e adaptado culturalmente (Duarte \& Bordin, 2000). Nesta pesquisa, estipulou-se como o critério de inclusão no grupo clínico para problema de comportamento as crianças que apresentaram em qualquer escala do teste (dividida em externalização, internalização e total) um escore limítrofe e/ ou clínico.

O RE-HSE-Pr, adaptado da versão para pais (Bolsoni-Silva \& Loureiro, 2009), descreve a frequência e topografia dos comportamentos presentes nas interações de professores e alunos, considerando respostas espontâneas do professor. Ele contempla 12 perguntasguia, que se desmembram em questões adicionais, sendo as respostas organizadas nas categorias denominadas de (1) Habilidades Sociais Educativas, (2) Práticas Educativas Negativas, (3) Habilidades Sociais Infantis e (4) Problemas Comportamentais. Na categoria de Habilidades sociais educativas encontram-se as seguintes subcategorias: (1) "Comunicando-se e negociando" referente às respostas como "conversar com aluno, acompanhar nas tarefas, elogiar"; (2) "Expressando sentimento e enfrentamento" em que se encontram comportamentos como "pede ajuda, pede desculpas, toca no aluno"; e (3) "Sentir-se bem" referente a relatos como "sente-se importante, seguro". Em Práticas Educativas Negativas há 3 subcategorias: (1) "Não habilidoso ativo" referente a respostas como "coloca o aluno para fora da sala de aula, pune, grita"; (2) "Não habilidoso passivo" relativo a comportamentos como "não fala nada, ignora comportamentos inadequado, sai da situação"; e (3) "Sente-se 
mal" em que se encontram relatos como "angustiado e cansado". Em relação aos comportamentos dos alunos, encontram-se as categorias Habilidades Sociais Infantis (desmembrada em (1) "Disponibilidade social e cooperação", em que há comportamentos como "faz elogios, obedece, presta atenção", e (2) "Expressando sentimento e enfrentamento" que se refere a relatos como "expressa opinião própria, explica porque agiu de determinada maneira") e Problemas de Comportamento (desmembrada em comportamentos externalizantes, tais como "bater, brigar, retrucar", e internalizantes, relativos a relatos como "isola-se, demonstra desinteresse, chora"). 0 instrumento foi empiricamente testado e encontra-se em fase de validação.

Por fim, o QRSH-Pr identifica as habilidades sociais das crianças, por meio de 24 afirmações, em uma escala de três pontos. O instrumento pode ser corrigido de diversas maneiras, mas optou-se por somar os escores. A seguir citam-se exemplos de afirmações que compõem o instrumento: "Faz pedidos?", "Procura sua atenção?", "Participa de grupos de jogos e trabalhos em sala de aula?". Bolsoni-Silva, Marturano e Loureiro (2009) analisaram as propriedades psicométricas do QRSH-Pr e classificaram a consistência interna e a validade preditiva como satisfatória.

\subsection{Procedimento de coleta e análise dos dados}

Num primeiro momento as pesquisadoras se preocuparam em obter a aprovação da Secretaria de Educação da cidade em que a pesquisa foi realizada e a aprovação do Comitê de Ética em Pesquisa da universidade em estava vinculado (2567/46/01/09). Na sequência, as escolas da rede pública de ensino de Educação Infantil (EI) e de Ensino Fundamental (EF) foram convidadas a participar da pesquisa. As professoras que aceitaram o convite assinaram o Termo de Consentimento Livre e Esclarecido (TCLE), e então foi solicitada a indicação de duas crianças: uma que consideravam apresentar problemas de comportamento, e outra que não apresentasse os mesmos tipos de comportamento. Então, para ambas as crianças, as professoras responderam aos instrumentos. Os dados foram categorizados conforme instruções próprias dos instrumentos e submetidos à análise estatística (SPSS). Foram conduzidos testes Qui-Quadrado e Teste $\mathrm{t}$ de Student, a fim de comparar os grupos quanto à escolaridade, classificação de grupo clínico ou não para problema de comportamento e sexo da criança. Nessas análises foram considerados $\mathrm{p}$ menor ou igual a 0,05, num intervalo de confiança de $95 \%$. Os resultados encontram-se em quatro tabelas, em que a primeira corresponde à caracterização da amostra e análise de variáveis categóricas. Nas outras tabelas apresentam-se as análises para ambas as escolarizações e, na sequência, têm-se os 
resultados dos subgrupos pré-escolares e escolares. Como devolutivas foram conduzidas palestras informativas sobre práticas educativas e comportamentos infantis, além de entregues, às escolas, cartilhas informativas sobre comportamentos infantis e práticas educativas (Bolsoni-Silva, 2008) e relatórios com os resultados da pesquisa resumidos.

\section{Resultados}

Na primeira seção apresentam-se a caracterização da amostra e a análise das variáveis categóricas (Tabela 1 ) e em seguida são descritos os resultados do Teste t de Student (Tabelas 2, 3 e 4).

Tabela 1

Tabulação cruzada da amostra em relação as variáveis sexo, escolaridade e problemas de comportamento, e Teste Qui-Quadrado

\begin{tabular}{clcccc}
\hline & & \multicolumn{2}{c}{ TRF } & \multicolumn{2}{c}{ Qui-Quadrado } \\
\cline { 3 - 6 } & & Clínico & Não Clínico & $\mathrm{X}^{2}$ & $p$ \\
\hline \multirow{2}{*}{ Sexo } & Menino & 115 & 54 & \multirow{2}{*}{43,58} & \multirow{2}{*}{0,00} \\
& Menina & 32 & 82 & & \\
\multirow{3}{*}{ Escolaridade } & Pré-escolar & 51 & 62 & \multirow{2}{*}{3,49} & 0,06 \\
& Escolar & 96 & 74 & & \\
\hline
\end{tabular}

Esses resultados demonstram na variável sexo uma relação significativa e na tabulação cruzada da amostra, observa-se que os meninos são a maioria no grupo de crianças indicadas como clínicas para problemas de comportamento. 
Tabela 2

Comparaçăo das categorias do RE-HSE-Pr e QRSH-PR entre as variáveis de problemas de comportamento, sexo e escolaridade

\begin{tabular}{|c|c|c|c|c|c|c|c|c|c|c|}
\hline \multirow{6}{*}{$\begin{array}{l}\text { RE-HSE-Pr: } \\
\text { Habilidades } \\
\text { sociais } \\
\text { educativas }\end{array}$} & \multirow[b]{4}{*}{$\begin{array}{l}\text { Expressando } \\
\text { sentimento e } \\
\text { enfrentamento }\end{array}$} & \multicolumn{2}{|c|}{ TRF } & \multicolumn{4}{|c|}{ Sexo } & \multicolumn{3}{|c|}{ Escolaridade } \\
\hline & & \multirow{2}{*}{$\begin{array}{l}\begin{array}{c}\text { Clínico } \\
(147)\end{array} \\
\text { Médi: }\end{array}$} & $\begin{array}{c}\text { Não } \\
\text { Clinico } \\
(136) \\
\end{array}$ & \multirow[b]{2}{*}{$p$} & $\begin{array}{c}\text { Menino } \\
(169)\end{array}$ & \multirow[t]{2}{*}{$\begin{array}{c}\text { Menina } \\
(114)\end{array}$} & & \multirow{2}{*}{$\begin{array}{c}\begin{array}{c}\text { Pré- } \\
\text { escolar } \\
(113)\end{array} \\
\text { Média }\end{array}$} & \multicolumn{2}{|l|}{$\begin{array}{l}\text { Escolar } \\
(170)\end{array}$} \\
\hline & & & (DP) & & Médi & & $p$ & & (DP) & $p$ \\
\hline & & - & - & - & - & - & - & $\begin{array}{c}3,98 \\
(2,47)\end{array}$ & $\begin{array}{c}2,49 \\
(2,28)\end{array}$ & 0,00 \\
\hline & Sentir-se bem & $\begin{array}{c}0,62 \\
(0,68)\end{array}$ & $0,91(0,75)$ & 0,00 & - & - & - & - & - & - \\
\hline & Total & - & - & - & - & - & - & $\begin{array}{l}15,84 \\
(4,54)\end{array}$ & $\begin{array}{c}14,6 \\
(4,88)\end{array}$ & 0,03 \\
\hline \multirow{4}{*}{$\begin{array}{l}\text { RE-HSE-Pr: } \\
\text { Práticas } \\
\text { Educativas } \\
\text { Negativas }\end{array}$} & $\begin{array}{c}\text { Não habilidoso } \\
\text { ativo }\end{array}$ & $\begin{array}{c}2,22 \\
(2,34)\end{array}$ & $\begin{array}{c}0,74 \\
(1,46)\end{array}$ & 0,00 & $\begin{array}{c}1,93 \\
(2,34)\end{array}$ & $\begin{array}{c}0,89 \\
(1,48)\end{array}$ & 0,00 & - & - & - \\
\hline & $\begin{array}{c}\text { Não habilidoso } \\
\text { passivo }\end{array}$ & $\begin{array}{c}0,15 \\
(0,46)\end{array}$ & $\begin{array}{c}0,05 \\
(0,25)\end{array}$ & 0,03 & $\begin{array}{c}0,14 \\
(0,44)\end{array}$ & $\begin{array}{c}0,05 \\
(0,26)\end{array}$ & 0,05 & - & - & - \\
\hline & Sentir-se mal & $\begin{array}{c}1,35 \\
(1,32)\end{array}$ & $\begin{array}{c}0,39 \\
(0,80)\end{array}$ & 0,00 & $\begin{array}{c}1,20 \\
(1,31)\end{array}$ & $\begin{array}{c}0,43 \\
(0,82)\end{array}$ & 0,00 & - & - & - \\
\hline & Total & $\begin{array}{c}3,73 \\
(3,33)\end{array}$ & $\begin{array}{c}1,18 \\
(1,90)\end{array}$ & 0,00 & $\begin{array}{c}3,27 \\
(3,29)\end{array}$ & $\begin{array}{c}1,37 \\
(2,09)\end{array}$ & 0,00 & - & - & - \\
\hline \multirow{3}{*}{$\begin{array}{l}\text { RE-HSE-Pr: } \\
\text { Habilidades } \\
\text { Sociais } \\
\text { Infantis }\end{array}$} & $\begin{array}{c}\text { Disponibilidade } \\
\text { social e } \\
\text { cooperação }\end{array}$ & $\begin{array}{c}6,71 \\
(3,53)\end{array}$ & $\begin{array}{c}9,36 \\
(3,92)\end{array}$ & 0,00 & $\begin{array}{c}7,25 \\
(3,74)\end{array}$ & $\begin{array}{c}9,08 \\
(4,00)\end{array}$ & 0,00 & - & - & - \\
\hline & $\begin{array}{l}\text { Expressando } \\
\text { sentimento e } \\
\text { enfrentamento }\end{array}$ & $\begin{array}{c}3,67 \\
(2,29)\end{array}$ & $\begin{array}{c}4,24 \\
(2,46)\end{array}$ & 0,05 & - & - & - & - & - & - \\
\hline & Total & $\begin{array}{l}10,43 \\
(4,97)\end{array}$ & $\begin{array}{l}13,64 \\
(4,93)\end{array}$ & 0,00 & $\begin{array}{l}11,08 \\
(5,03)\end{array}$ & $\begin{array}{l}13,29 \\
(5,17)\end{array}$ & 0,00 & - & - & - \\
\hline \multirow{3}{*}{$\begin{array}{l}\text { RE-HSE-Pr: } \\
\text { Problemas de } \\
\text { Comportame } \\
\text { nto }\end{array}$} & $\begin{array}{c}\text { Problemas } \\
\text { externalizantes }\end{array}$ & $\begin{array}{c}6,93 \\
(5,84)\end{array}$ & $\begin{array}{c}1,79 \\
(3,18)\end{array}$ & 0,00 & $\begin{array}{c}5,95 \\
(5,77)\end{array}$ & $\begin{array}{c}2,26 \\
(3,87)\end{array}$ & 0,00 & - & - & - \\
\hline & $\begin{array}{c}\text { Problemas } \\
\text { internalizantes }\end{array}$ & $\begin{array}{c}3,18 \\
(3,31)\end{array}$ & $\begin{array}{c}1,35 \\
(2,24)\end{array}$ & 0,00 & $\begin{array}{c}2,65 \\
(3,07)\end{array}$ & $\begin{array}{c}1,79 \\
(2,79)\end{array}$ & 0,02 & - & - & - \\
\hline & Total & $\begin{array}{l}10,89 \\
(7,50)\end{array}$ & $\begin{array}{c}3,24 \\
(4,74)\end{array}$ & 0,00 & $\begin{array}{c}9,17 \\
(7,73)\end{array}$ & $\begin{array}{c}4,32 \\
(5,75)\end{array}$ & 0,00 & - & - & - \\
\hline \multicolumn{2}{|c|}{ QRSH-Pr } & $\begin{array}{c}29,76 \\
(11,55)\end{array}$ & $\begin{array}{l}41,06 \\
(7,50)\end{array}$ & 0,00 & $\begin{array}{c}32,80 \\
(11,22)\end{array}$ & $\begin{array}{c}38,75 \\
(10,52)\end{array}$ & 0,00 & - & - & - \\
\hline
\end{tabular}

Verifica-se na Tabela 2 diferenças significativas entre os grupos clínico e não clínico para problemas de comportamento, sendo que crianças clínicas apresentaram maiores médias nas categorias e subcategorias de Práticas Educativas Negativas e Problemas de Comportamento. Aquelas indicadas como não clínicas apresentaram maiores médias nas Habilidades Sociais. Logo, crianças clínicas para problemas de comportamento apresentaram maiores frequências de comportamentos problema e práticas educativas negativas, e menores frequências de habilidades sociais. Na comparação entre sexos, é possível observar diferenças estatísticas nas categorias de Prática educativa negativa, Habilidades sociais infantis e Problemas de Comportamento. O grupo dos meninos apresentou maiores médias nas categorias de problemas de comportamento e práticas educativas negativas, e menores médias nas habilidades sociais. $E$ entre os grupos de crianças pré-escolares e escolares, verificou-se diferença na categoria de Habilidades Sociais Educativas, indicando professores parecem ser mais habilidosos com crianças pré-escolares. A seguir, 
apresentam-se os resultados dos alunos pré-escolares (Tabela 3) e escolares (Tabela 4), de acordo com as categorias do RE-HSE-Pr e QRSH-Pr.

Tabela 3

Comparação das categorias do RE-HSE-Pr e QRSH-Pr, dos alunos pré-escolares, entre as variáveis de problemas de comportamento e sexo

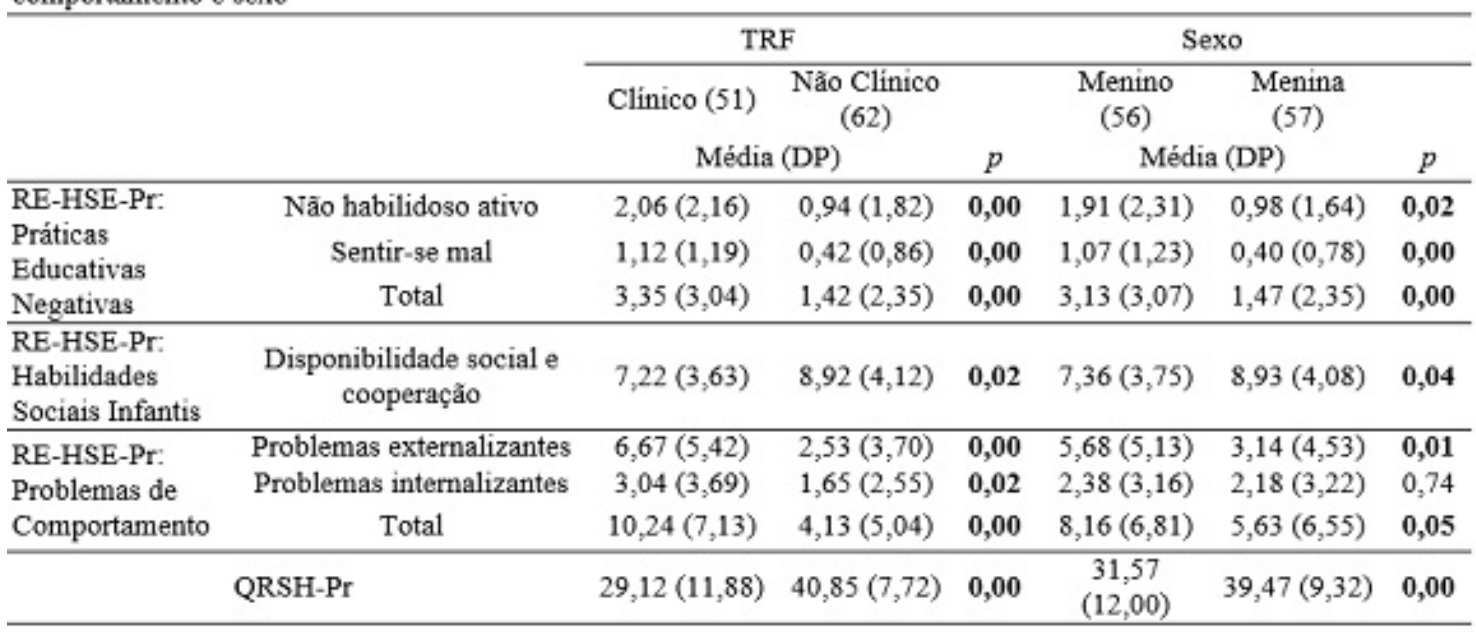

$\mathrm{Na}$ Tabela 3, vericaram-se diferenças significativas entre os grupos, demonstrando que professores parecem ser menos habilidosos com crianças clínicas e meninos para problemas de comportamento, que por sua vez apresentaram maiores médias de problemas comportamentais e menores médias de habilidades sociais. Já na Tabela 4 abaixo, observa-se que crianças escolares indicadas como clínicas e meninos aparentam possuir maior frequência de problemas de comportamento, menor frequência de habilidades sociais, e seus professores aparentam ser menos habilidosos. 
Tabela 4

Comparação das categorias do RE-HSE-Pr e QRSH-PR, dos alunos escolares, entre as variáveis de problemas de comportamento e sexo

\begin{tabular}{|c|c|c|c|c|c|c|c|}
\hline & & \multicolumn{2}{|c|}{ TRF } & \multicolumn{4}{|c|}{ Sexo } \\
\hline & & & \multirow{2}{*}{$\begin{array}{l}\text { Não Clínico } \\
(74) \\
\text { (DP) }\end{array}$} & \multirow[b]{2}{*}{$p$} & $\begin{array}{l}\text { Menino } \\
(113)\end{array}$ & $\begin{array}{c}\text { Menina } \\
(57)\end{array}$ & \multirow[b]{2}{*}{$p$} \\
\hline & & Média (DP) & & & \multicolumn{2}{|c|}{ Média (DP) } & \\
\hline $\begin{array}{l}\text { Habilidades } \\
\text { sociais } \\
\text { educativas }\end{array}$ & Sentir-se bem & $0,5(0,60)$ & $0,95(0,72)$ & 0,00 & - & - & - \\
\hline \multirow{4}{*}{$\begin{array}{l}\text { RE-HSE-Pr: } \\
\text { Práticas } \\
\text { Educativas } \\
\text { Negativas }\end{array}$} & Não habilidoso ativo & $2,31(2,44)$ & $0,57(1,06)$ & 0,00 & $1,94(2,37)$ & $0,79(1,31)$ & 0,00 \\
\hline & Não habilidoso passivo & - & - & - & $0,13(0,41)$ & $0,02(0,13)$ & 0,01 \\
\hline & Sentir-se mal & $1,47(1,37)$ & $0,36(0,75)$ & 0,00 & $1,26(1,35)$ & $0,46(0,87)$ & 0,00 \\
\hline & Total & $3,93(3,47)$ & $0,97(1,40)$ & 0,00 & $3,34(3,41)$ & $1,26(1,82)$ & 0,00 \\
\hline \multirow{3}{*}{$\begin{array}{l}\text { RE-HSE-Pr: } \\
\text { Habilidades } \\
\text { Sociais Infantis }\end{array}$} & $\begin{array}{l}\text { Disponibilidade social e } \\
\text { cooperação }\end{array}$ & $6,45(3,47)$ & $9,73(3,72)$ & 0,00 & $7,19(3,76)$ & $9,23(3,94)$ & 0,00 \\
\hline & $\begin{array}{l}\text { Expressando sentimento e } \\
\text { enfrentamento }\end{array}$ & $3,42(2,32)$ & $4,18(2,48)$ & 0,04 & $3,48(2,36)$ & $4,28(2,45)$ & 0,04 \\
\hline & Total & $9,91(4,85)$ & $13,97(4,73)$ & 0,00 & $10,73(5,07)$ & $13,56(4,95)$ & 0,00 \\
\hline \multirow{4}{*}{$\begin{array}{l}\text { RE-HSE-Pr: } \\
\text { Problemas de } \\
\text { Comportamento }\end{array}$} & Problemas externalizantes & $7,07(6,07)$ & $1,18(2,53)$ & 0,00 & $6,08(6,07)$ & $1,39(2,86)$ & 0,00 \\
\hline & Problemas internalizantes & $3,26(3,11)$ & $1,11(1,93)$ & 0,00 & $2,79(3,03)$ & $1,40(2,25)$ & 0,00 \\
\hline & Total & $11,24(7,70)$ & $2,50(4,38)$ & 0,00 & $9,67(8,13)$ & $3,00(4,49)$ & 0,00 \\
\hline & QRSH-Pr & $30,11(11,41)$ & $41,23(7,37)$ & 0,00 & $\begin{array}{c}33,41 \\
(10,81)\end{array}$ & $\begin{array}{c}38,02 \\
(11,64)\end{array}$ & 0,01 \\
\hline
\end{tabular}

Em suma, crianças escolares e pré-escolares indicadas como clínicas para problemas de comportamento apresentaram maiores frequências de comportamentos problema e práticas educativas negativas e menores frequências de habilidades sociais. O grupo dos meninos apresentou maiores médias nas categorias de problemas de comportamento e de práticas educativas negativas, e menores médias de habilidades sociais. Crianças pré-escolares apresentaram maiores médias nas categorias de habilidades sociais e habilidades sociais educativas, quando comparadas com as crianças escolares. Mas, nas interações com crianças pré-escolares indicadas como clínicas para problemas de comportamento e do sexo masculino os professores parecem ser menos habilidosos, sendo que essas crianças também apresentaram maiores médias de problemas comportamentais e menores médias de habilidades sociais. De maneira semelhante, crianças escolares indicadas como clínicas e do sexo masculino apresentaram maior frequência de problemas comportamentais internalizantes e externalizantes, menor frequência de habilidades sociais, e, seus professores relataram menos habilidades sociais educativas. 


\section{Discussão}

\subsection{Os diferentes sexos e os comportamentos das crianças e professores}

Os achados desta pesquisa demonstram que há diferenças significativas entre meninos e meninas nos comportamentos infantis e nas práticas educativas de professores, tanto com crianças préescolares, como escolares. Na comparação dos comportamentos infantis, constatou-se que meninos apresentaram escores mais baixos de habilidades sociais e mais altos de problemas de comportamento, tanto internalizantes como externalizantes. Esses resultados corroboram os resultados de Pizato, Marturano e Fontaine (2014) e Zhang e Sun (2011) que também evidenciaram nas meninas altos índices de habilidades sociais, e baixos índices de problemas de comportamento, tando internalizantes, como externalizantes. No entanto, esse resultado se diferencia, em parte, de outros estudos (como Baker, 2006, Henricsson e Rydell, 2006 e Silver et al., 2010) em que meninos apresentaram altos índices de comportamentos externalizantes, e as meninas comportamentos internalizantes. Tais discrepâncias podem ser explicadas devido as diferentes metodologias de pesquisa e em especial aos diferentes usos de instrumentos para coleta de dados. Henricsson e Rydell (2006), por exemplo, utilizaram além de instrumentos validados (CBQ de Rutter, Tizard e Whitmore, 1970, e STRS do Pianta, 1996), observações diretas no ambiente escolar (Henricsson \& Rydell, 2004). Outra hipótese baseia-se nas diferentes possibilidades de utilização dos instrumentos, já que eles podem ser utilizados como rastreadores ou diagnósticos (como é o caso do TRF). Assim, parece que metodologias de pesquisa que associam diferentes tipos de instrumentos (testes validados e observações no ambiente natural do participante) produzem resultados mais específicos sobre 0 fenômeno. Por fim, discute-se a respeito da carência de instrumentos de avaliação comportamental padronizados, validados e empiricamente testados para a cultura brasileira, a fim de auxiliar na avaliação e diagnóstico de diferentes aspectos da saúde mental de crianças, bem como para o planejamento de ações interventivas (preventivas ou não) que sejam eficazes e efetivas (Duarte \& Bordin, 2000; Pesce, 2009).

Em relação ao dado referente às crianças com altos escores de habilidades sociais e baixos índices de problemas de comportamento, também evidenciado em outros estudos (Baker et al., 2008; BolsoniSilva et al., 2010; Henricsson \& Rydell, 2006; Pizato et al., 2014), pode indicar que padrões comportamentais problemáticos concorrem com habilidades sociais, considerando que a aprendizagem de comportamentos problema tende a prejudicar a aquisição de 
habilidades (Bolsoni-Silva \& Carrara, 2010; Bolsoni-Silva \& Marturano, 2002; Patterson et al., 2000).

Dentre os comportamentos dos educadores, notou-se que professores são menos habilidosos com os meninos, o que demonstra consistência com estudos de Baker et al. (2008), Henricsson e Rydell (2006), Silver et al. (2010) e Zhang e Sun (2011), em que houveram maiores relacionamentos de conflito e raiva mútua entre professores e alunos do sexo masculino. Observa-se, assim, que os meninos parecem emitir mais problemas de comportamento, já que ingressam na escola com mais energia, entusiasmo e agressidade (Baker, 2006), e professores, por sua vez, respondem de maneira pouco habilidosa. Para Doumen et al. (2008) esse fenômeno ocorre devido a influência mútua de comportamentos agressivos das interações, podendo intensificar os padrões de problemas de comportamento externalizante no decorrer da escolarização, e problemas de saúde nos professores, como por exemplo burnout (Pas et al., 2010). Nesse mesmo sentido, tem-se a diferença na sub-categoria "Sentir-se mal", de Práticas Educativas Negativas, entre os sexos de crianças préescolares e escolares, com maiores médias atribuídas aos meninos. Percebe-se, assim, que nas interações entre professores e alunos do sexo masculino a produção de sentimentos como estresse, preocupação, nervosismo. Essas discrepâncias de comportamentos entre os sexos também podem ser explicadas pelas influências culturais no estabelecimento e manutenção dos comportamentos, além das variáveis filogenéticas e ontogenéticas, conforme aponta Skinner (2009). A escola, como a família, exerce um papel de socialização em que as crianças aprendem os papéis de gêneros que a cultura considera apropriada para cada sexo (Bee \& Boyd, 2011). Logo, as crianças são estimuladas de diferentes maneiras e aprendem comportamentos, interesses e habilidades conforme a cultura em que se insere, ao se apropriarem dos gêneros masculino ou feminino (Bee $\&$ Boyd, 2011).

\subsection{As diferentes escolaridades e os comportamentos de crianças e professores}

A comparação das práticas educativas nos dois momentos de escolarizações (pré-escolares e escolares), demonstra que professores parecem mais habilidosos com crianças da pré-escola: professores relataram emitir mais habilidades sociais educativas na pré-escola, ainda que a frequencia de práticas negativas não se diferenciou. O resultado está em concordância com estudos internacionais (Baker, 2006; Buyse et al., 2008; Hamre \& Pianta, 2005) que constataram uma tendência à diminuição de relacionamentos positivos entre professores e alunos ao longo da escolarização, em paralelo a progressão de problemas 
comportamentais. Parece, portanto, que conforme a criança vai evoluindo nos níveis educacionais, os ambientes vão se tornando mais complexos e exigentes, nos âmbitos acadêmico e social (Buyse et al., 2008; Hamre \& Pianta, 2005). E em situações sociais complexas, crianças que apresentam problemas de comportamento tendem a emiti-los em maior frequência provocando confrontos (Buyse et al., 2008), já que não possuem em seu repertório outros comportamentos. Aliás, considerando a legislação brasileira, esse fenômeno pode ser explicado diferentes organizações e planejamentos dos parâmetros curriculares, em que na pré-escola (EI) tem-se um espaço lúdico e propício para as brincadeiras (MEC, 2009), enquanto que o período escolar (EF) enfatiza a alfabetização e o letramento em um enquadramento mais rígido (MEC, 2010). O destaque para as atividades acadêmicas e a diminuição de atividades lúdicas no $\mathrm{EF}$, tende a exigir maior variabilidade de comportamentos das crianças, podendo produzir situações de conflito (Bolsoni-Silva et al., 2010; Castro \& Bolsoni-Silva, 2008).

\subsection{Diferenças entre grupos clínico e não clínico para problemas de comportamento e os comportamentos de crianças e professores}

Os resultados indicam uma semelhança nas frequências de comportamentos categorizados como Habilidades Sociais Educativas entre crianças clínicas e não clínicas para problemas de comportamento. Nas Práticas educativas negativas, observou-se diferenças com maiores médias nas crianças clínicas. Assim, professores parecem interagir com alunos clínicos para problemas comportamentais, em ambas escolaridades, de maneira mais punitiva e conflituosa e, assim, tais interações não produzem consequências prazeirosas para educadores (justificando a diferença na sub categoria "sentir-se bem"). Relações negativas entre alunos com problemas de comportamento e professores demonstram consistência com a literatura internacional e são preocupantes considerando que os impactos dessas interações se associam com fracasso acadêmico e social e indicam estabilidade e escalada de problemas de comportamento (Baker, 2006; Baker et al., 2008; Henricsson \& Rydell, 2006; Doumen et al., 2008; O'Connor et al., 2011; Silver et al., 2010; Zhang \& Sun, 2011). Dessa forma, quando a criança ingressa na escola e apresenta em seu repertório comportamentos agressivos, inicia-se um processo de fortalecimento e ampliação de relações conflituosas que tendem a produzir prejuízos ao longo da vida (Doumen et al., 2008; Granic \& Patterson, 2006; O'Connor et al., 2011; Patterson et al., 1992). De maneira semelhante, pesquisas (Bolsoni-Silva \& Mariano, 2014; Castro \& Bolsoni-Silva, 2008) com a população brasileira, evidenciaram a utilização de práticas educativas 
negativas e comportamentos punitivos, em detrimento daqueles assertivos e reforçadores, influenciadas por uma cultura coercitiva (Castro \& Bolsoni-Silva, 2008; Viecili \& Medeiros, 2002). A utilização de punição, ainda que suprima o comportamento considerado inadequado, produz também efeitos colaterais, podendo intensificar os problemas (Sidman, 1995; Viecili \& Medeiros, 2002; Zanotto, 2000). Em relação as Habilidades sociais educativas, Bolsoni-Silva e Mariano (2014), numa amostra reduzida, também identificaram que professoras interagem quantitativamente de maneira semelhante com alunos clínicos e não clínicos para problemas, mas qualitativamente é possivel observar diferenças nessas interações. No grupo de crianças clínicas para problemas comportamentais, da pesquisa supracitada, o conteúdo das interações, ainda que emitido de maneira habilidosa, associa-se aos problemas e não ocorre em outros momentos e sobre outros assuntos. A conversa contingente ao comportamento problema pode ser reforçador para a criança, produzindo a manutenção deste comportamento, já que o professor, inadvertidamente, oferece atenção social contingente ao comportamento inadequado e não o faz com a mesma frequencia e intensidade diante de comportamentos adequados (Bolsoni-Silva \& Mariano, 2014). Já no grupo não clínico para problemas, observou-se que os professores também conversam quando as crianças emitem comportamentos externalizantes, mas também o fazem em outros contextos: quando as crianças são habilidosas e diante de assuntos de interesses das mesmas. Assim, o comportamento habilidoso do professor quando contingente somente aos comportamentos problemáticos dos alunos podem manter comportamentos inadequados, ao invés de extingui-los (Bolsoni-Silva \& Mariano, 2014). Outros autores (Del Prette \& Del Prette, 2005; Marturano \& Loureiro, 2003) também discutiram que as interações que ocorrem na escola podem estabelecer e/ou manter problemas de comportamento e déficits nas habilidades sociais devido a má administração de recompensas e punições. Desta forma, parece que professores apresentam dificuldades em lidar com alunos com problemas comportamentais, de modo que tal deficiência além de reforçar padrões problemáticos, não promovem o ensino de modelos alternativos e mais adaptativos aos alunos, ainda que de maneira não intencional (Castro \& Bolsoni-Silva, 2008; Del Prette \& Del Prette, 2005). Por fim, destaca-se, de maneira semelhante aos resultados de estudos empíricos internacionais (Baker, 2006; Baker et al., 2008; Doumen et al., 2008; O'Connor et al., 2011; Silver et al., 2010), que professores tendem a ser mais próximos, comunicativos e afetuosos com crianças não clínicas para problemas de comportamento. Contudo, parece que ao aumentar a proximidade, comunicação e afeto e, em contrapartida, diminuir interações conflituosas com alunos, professores podem influenciar positivamente na trajetória de 
desenvolvimento destes, promovendo um efeito compensatório para problemas de comportamento (Baker et al., 2008; Silver et al., 2010).

Já em referencia às diferenças nas habilidades sociais entre crianças clínicas e não clínicas para problemas de comportamento, observa-se nas crianças clínicas escores inferiores de habilidades sociais, que é bastante semelhante com a literatura (crianças com comportamentos problemas apresentam deficits de aprendizagem de habilidades sociais) (Baker et al., 2008; Bolsoni-Silva et al., 2010; Henricsson \& Rydell, 2006; Silver et al., 2010). No entanto, a habilidade social atua como fator de proteção ao desenvolvimento infantil, previnindo o estabelecimento, manutenção e escalada de problemas de comportamento (Baker, 2006; Bolsoni-Silva et al., 2010; Henricsson $\&$ Rydell, 2006; Pizato et al., 2014).

Finalizando, discute-se que tais resultados podem subsidiar 0 planejamento de programas preventivos e interventivos para crianças com problemas de comportamento, sugerindo o desenvolvimento de relações positivas, afetuosas e caracterizadas pela proximidade (em oposição às relações conflituosas e punitivas). Isto porque Habilidades sociais educativas parecem promover a aprendizagem de habilidades sociais infantis, por meio de procedimentos como modelação (imitação), modelagem (reforço diferencial), e emissão de feedback eficaz (Baker et al., 2008), que, então, poderão concorrer com problemas de comportamento. Dessa forma, as Habilidades sociais educativas parecem impactar indiretamente nos problemas de comportamento, enquanto que práticas negativas impactam de maneira direta, considerando que respostas agressivas de professores promovem a manutenção, podendo intensificar, padrões de comportamentos agressivos (Doumen et al., 2008; O'Connor et al., 2011). Mas, para que professores sejam socialmente competentes, não bastaria a emissão de determinados comportamentos, pois é preciso considerar o aspecto funcional presente nas interações (Del Prette \& Del Prette, 2008).

\section{Considerações finais}

O presente estudo apresenta uma limitação relacionada à fonte única de coleta de dados, pois somente os professores responderam sobre os próprios comportamentos, e sobre os comportamentos dos alunos. Apesar da limitação citada, esse estudo possui uma amostra representativa, o controle de múltiplas variáveis e a utilização de instrumentos empiricamente testados. Este estudo avança em relação às pesquisas brasileiras anteriores já que elas ora avaliaram somente os comportamentos externalizantes e internalizantes das crianças, ora avaliaram somente os professores, ou apesar de avaliarem os 
comportamentos presentes nas interações destes apresentaram uma amostra reduzida. Futuras pesquisas poderiam expandir a amostra, selecionando crianças de outros e diferentes contextos (comparar cidades, escolas públicas e privadas), propor metodologias diversas (incluir observações diretas no ambiente e ampliar a fonte de coleta de dados) e padronizar e validar instrumentos de avaliação comportamental.

\section{Referências}

Achenbach, T. M., \& Edelbrock, C. S. (1978). The classification of child psychopathology: a review and analysis of empirical efforts. Psychological Bulletin, 85(6), 1275-1301.

Achenbach, T. M., \& Rescorla, L. A. (2001). Manual for the ASEBA school-age forms, profiles. Burlington, VT: University of Vermont, Research Center for Children, Youth, Families.

Baker, J. A. (2006). Contributions of teacher-child relationships to positive school adjustment during elementary school. Journal of School Psychology, 44(3), 211-229.

Baker, J. A., Grant, S., \& Morlock, L. (2008). The teacher-student relationship as a developmental context for children with internalizing or externalizing behavior problems. School Psychology Quarterly, 23(1), 3-15.

Bee, H., \& Boyd, B. (2011). A criança em desenvolvimento (12a ed.). Porto Alegre: Artmed.

Bolsoni-Silva, A. T., \& Marturano, E. M. (2002). Práticas educativas parentais e problemas de comportamento: uma análise à luz das habilidades sociais. Estudos de Psicologia, 7(2), 227-235.

Bolsoni-Silva, A. T. (2008). Práticas educativas: manejo comportamental e comportamentos pró-sociais. In: Capellini, V. L. M. F. (Org.). Práticas em educação especial inclusiva na área da deficiência mental ( $2 a$ ed.). Bauru: MEC/FC/SEE. Disponível em: http://www.rihs.ufscar.br/.

Bolsoni-Silva, A. T., Marturano, E. M., \& Loureiro, S. R. (2009). Contribuições para a construção e validação do questionário de respostas socialmente habilidosas: versão para professores QRSH-PR. Spanish Journal of Psychology, 12(2), 349-359.

Bolsoni-Silva, A. T., Marturano, E. M., \& Freiria, R. L. B. (2010). Indicativos de problemas de comportamento e de habilidades sociais em crianças: um estudo longitudinal. Psicologia: Reflexão e Crítica, 23(3), 506-515.

Bolsoni-Silva, A. T., \& Loureiro, S. R. (2009). Validação do roteiro de entrevista de Habilidades Sociais Educativas Parentais (RE-HSEP). Avaliação Psicológica, 9(1), 63-75. 
Bolsoni-Silva, A. T., \& Carrara, K. (2010). Habilidades sociais e análise do comportamento: compatibilidades e dissensões conceitual-metodológicas. Psicologia em Revista (Online), 16(2), 330-350.

Bolsoni-Silva, A. T., \& Mariano, M. L. (2014). Práticas educativas de professores e comportamentos infantis, na transição ao primeiro ano do Ensino Fundamental. Estudos e Pesquisas em Psicologia, 14(3), 834-853.

Bolsoni-Silva, A. T., Mariano, M. L., Loureiro, S. R., \& Bonaccorsi, C. (2013). Contexto escolar: práticas educativas do professor, comportamento e habilidades sociais infantis. Revista Semestral da Associação Brasileira de Psicologia Escolar e Educacional, 17(2), 259-269.

Brasil. Ministério da Educação (MEC). (2009). Diretrizes Curriculares Nacionais para a Educação Infantil. Resolução CNE/CEB 5/2009. Diário Oficial da União, Brasília, 18 de dezembro de 2009.

Brasil. Ministério da Educação (MEC). (2010). Diretrizes Curriculares Nacionais para o Ensino Fundamental. Resolução CNE/CEB 7/2010. Diário Oficial da União, Brasília, 15 de dezembro de 2010.

Buyse, E., Verschueren, K., Doumen, S., Damme, J. V., \& Maes, F. (2008). Classroom problem behavior and teacher-child relationships in kindergarten: the moderating role of classroom climate. J ournal of School Psychology, 46(4), 367-391.

Castro, A. B., \& Bolsoni-Silva, A. T. (2008). Habilidades sociais na educação: relações entre concepções e práticas docentes na educação infantil. In: Capellini, V. L. F., \& Manzoni, R. M. (Org.). Políticas públicas, práticas pedagógicas e ensinoaprendizagem: diferentes olhares sobre o processo educacional. São Paulo: Cultura Acadêmica.

Del Prette, Z. A. P., \& Del Prette, A. (2005). Psicologia das habilidades sociais na infância: teoria e prática. Petrópolis: Vozes.

Del Prette, Z. A. P., \& Del Prette, A. (2008). Um sistema de categorias de habilidades sociais educativas. Paidéia, 18(41), 517-530.

Doumen, S., Verschueren, K., Buyse, E., Germeijs, V., Luyckx, K., \& Soenens, B. (2008). Reciprocal relations between teacher-child conflict and aggressive behavior in kindergarten: a three-wave longitudinal study. Journal of Clinical Child and Adolescent Psychology, 37(3), 588-599.

Duarte, C. S., \& Bordin, I. A. S. (2000). Instrumentos de avaliação. Revista Brasileira de Psiquiatria, 22(Suppl. 2), 55-58.

Granic, I., \& Patterson, G. (2006). Toward a comprehensive model of antisocial development: a dynamic systems approach. Psychological Review, 113(1), 101-131. 
Gonçalves, E., \& Murta, S. (2008). Avaliação dos efeitos de uma modalidade de treinamento de habilidades sociais para crianças. Psicologia: Reflexão e Crítica, 21(3), 430-436.

Hamre, K. H., \& Pianta, R. C. (2005). Can instructional and emotional support in the first-grade classroom make a difference for children at risk of school failure? Child Development, 76(5), 949-967.

Henricsson, L., \& Rydell, A. (2004). Elementary school children with behaviour problems: teacher-child relations and selfperception. A prospective study. Merrill Palmer Quarterly, 50: 111-138.

Henricsson, L., \& Rydell, A. (2006). Children with behaviour problems: the influence of social competence and social relations on problem stability, school achievement and peer acceptance across the first six years of school. Infant and Child Development, 15(4), 347-366.

Lyra, G. F. D., Assis, S. G., Njaine, K., Oliveira, R. V. C., \& Pires, T. O. (2009). A relação entre professores com sofrimento psíquico e crianças escolares com problemas de comportamento. Ciência \& Saúde Coletiva, 14(2), 435-444.

Mariano, M. L. (2011). Análise de práticas educativas de professores na interação com alunos com e sem problemas de comportamentos. Dissertação de Mestrado, Pós-Graduação em Psicologia do Desenvolvimento e Aprendizagem. Universidade Estadual Paulista, Campus Bauru.

Marturano, E. M., \& Loureiro, S. R. (2003). O desenvolvimento socioemocional e as queixas escolares. In Del Prette, A., \& Del Prette, Z. A. P. (Orgs.) (2007). Habilidades sociais, desenvolvimento e aprendizagem: questões conceituais, avaliação e intervenção (pp. 259-291). Campinas, Alínea (2 ed.).

O'Connor, E. E., Dearing, E., \& Collins, B. A. (2011). Teacher-child relationship and behavior problem trajectories in elementary school. American Educational Research Journal, 48(1), 120162.

Pas, E. T., Bradshaw, C. P., Hershfeldt, P. A., \& Leaf, P. J. (2010). A multilevel exploration of the influence of teacher efficacy and burnout on response to student problem behavior and schoolbased service use. School Psychology Quarterly, 25(1), 13-27.

Patterson, G., Reid, J., \& Dishion, T. (1992). Antisocial boys (4a ed.), Eugene: Castalia.

Patterson, G. R., Degarmo, D. S., Knutson, N. (2000). Hyperactive and antisocial behaviors: comorbid or two points in the same process? Development and Psychopathology, 12(1), 91-106. 
Pedrini, J. R., \& Frizzo, G. B. (2010). Avaliação de indicadores de problemas de comportamento infantil relatados por pais e professores. Aletheia, (33), 69-83.

Pereira, M. T., Marturano, E. M., Gardinal-Pizato, E. C., \& Fontaine, A. M. G. (2011). Possíveis contribuições da educação infantil para o desempenho e a competência social de escolares. Revista Semestral da Associação Brasileira de Psicologia e Educacional, 15(1), 101-109.

Pianta, R. C. (1996). Manual and scoring guide for the studentteacher relationship scale. University of Virginia: Charlottesville, VA.

Picado, J. R., \& De Rose, T. M. S. (2009). Acompanhamento de préescolares agressivos: adaptação na escola e relação professoraluno. Psicologia Ciência e Profissão, 29(1), 132-145.

Pizato, E. C. G., Marturano, E. M., \& Fontaine, A. M. G. V. (2014). Trajetórias de habilidades sociais e problemas de comportamento no ensino fundamental: influência da educação infantil. Psicologia: Reflexão e Crítica, 27(1), 189-197.

Pesce, R. (2009). Violência familiar e comportamento agressivo e transgressor na infância: uma revisão da literatura. Ciência: saúde coletiva, 14(2), 507-518.

Rutter M., Tizard, J., \& Whitmore, K. (1970). Education, Health and Behaviour. Longman Group, Ltd.: London.

Saud, L. F., \& Tonelotto, J. M. F. (2005). Comportamento social na escola: diferenças entre gênero e séries. Psicologia Escolar e Educacional, 9(1), 47-57.

Sidman, M. (1995). Coerção e suas implicações. Campinas, SP: Editora Psy.

Silver, R. B., Jeffrey, R. M, Jeffrey, M. A., \& Marilyn, J. E. (2010). The impact of parents, child care providers, teachers, and peers on early externalizing trajectories. Journal of School Psychology, 48(6), 555-583.

Skinner, B. F. (2009). Ciência e Comportamento Humano (11a ed.). Brasília: Ed. UnB/FUNBEC.

Viecili, J., \& Medeiros, J. G. (2002). A coerção e suas implicações na relação professor-aluno. Psico-UFS, 7(2), 229-238.

Zanotto, M. L. B. (2000). Formação de professores: a contribuição da análise do comportamento. São Paulo, SP: EDUC.

Zhang, X., \& Sun, J. (2011). The reciprocal relations between teachers' perceptions of children's behavior problems e teacher-child relationships in the first preschool year. The Journal of Genetic Psychology: Research e Theory on Human Development, 172(2), 176-198. 


\section{Endereço para correspondência}

\section{Marília Mariano}

Universidade Estadual Paulista "Júlio de Mesquita Filho"

Av. Engenheiro Luiz Edmundo Carrijo Coube, CEP 17033-360, Bauru - SP, Brasil

Endereço eletrônico: mariano.mrl@gmail.com

\section{Alessandra Turini Bolsoni-Silva}

Universidade Estadual Paulista "Júlio de Mesquita Filho"

Av. Engenheiro Luiz Edmundo Carrijo Coube, CEP 17033-360, Bauru - SP, Brasil

Endereço institucional: bolsonisilva@gmail.com

Recebido em: 02/10/2015

Reformulado em: 07/01/2016

Aceito para publicação em: 14/01/2016

\section{Notas}

* Mestre em Psicologia do Desenvolvimento e Aprendizagem da Faculdade de Ciências, Unesp, Bauru.

** Livre Docente em Psicologia Clínica, docente do PPG Psicologia do Desenvolvimento e Aprendizagem e Depto de Psicologia, da Faculdade de Ciências, Unesp, Bauru. 